\title{
A Note on Skew Energy of Hadamard Graph
}

\author{
M. Saravanan, K. M. Kathiresan
}

\begin{abstract}
The skew spectrum and skew energy of an oriented graph $G^{\sigma}$ are respectively the set of eigenvalues of the adjacency matrix of $G^{\sigma}$ and the sum of the absolute values of the eigen values of the adjacency matrix of $G$. In this work, we find and study the skew spectrum and the skew energy of Hadamard graph for a particular orientation.
\end{abstract}

Keywords : Hadamard Graph, eigen values, skew energy.

\section{INTRODUCTION}

Let $\mathrm{G}$ be a undirected simple graph of order $n$ with vertex set $V=V(G)=\left\{v_{1}, v_{2}, \ldots, v_{n}\right\} \quad$ and edge set $E=E(G)$. The adjacency matrix of $\mathrm{G}$ is the $n \times n$ matrix $A(G)=\left[a_{i j}\right]$, is defined as $a_{i j}=1$ if $v_{i} v_{j}$ is an edge and 0 otherwise. All eigenvalues of $A(G)$ are real, since $A(G)$ is real and symmetric.. The energy $\varepsilon(G)$, of a graph $G$ is defined to be the sum of the absolute values of its eigen values. Hence if $A(G)$ is the adjacency matrix of $G$ and $\lambda_{1}, \lambda_{2}, \cdots, \lambda_{p}$ are the eigen values of $A(G)$, then $\varepsilon(G)=$ $\sum_{i=1}^{p}\left|\lambda_{i}\right|$. The set $\left\{\lambda_{1}, \lambda_{2}, \cdots, \lambda_{p}\right\}$ is called as the spectrum of $G$, denoted by $\operatorname{Spec}(G)$. Suppose $\theta_{1}, \theta_{2}, \cdots, \theta_{k}$ are the distinct eigen values of $G$ and $m_{i}$ is the multiplicity of $\theta_{i}(i=1,2, \ldots, k)$, then the spectrum can be written as the multiset $\left\{\left[\theta_{1}\right]^{m_{1}},\left[\theta_{2}\right]^{m_{2}}, \cdots,\left[\theta_{k}\right]^{m_{k}}\right\}$. For more on spectrum and energy of graphs, we refer [5], [7], [9], [11].

If $G$ is a connected graph and $x$ is a vertex of $G$, then $N_{i}(x)$ is the set of vertices of $G$ which are at distance $i$ from the vertex $x$. For $i=1$, we get $N_{i}(x)=N(x)$. A $k$ - regular connected graph $G$ with diameter $d$ is called distance regular[6], if there are nonnegative integers $b_{i}, c_{i}(i=0,1, \ldots, d)$ such that for every $x, y \in V(G)$ at distance $i$, there are exactly $b_{i}$ neighbors of $y$ in $N_{i+1}(x)$ and $c_{i}$ neighbors of $y$ in $N_{i-1}(x)$.

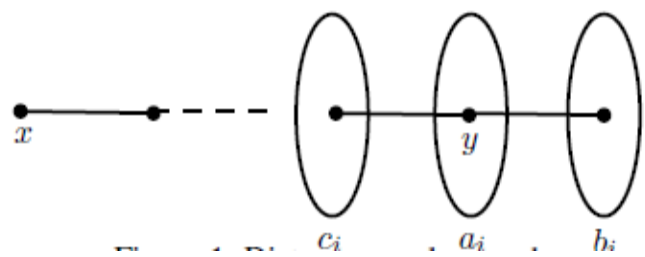

Fig.1. Distance Regular Graph

The sequence $\left\{b_{0}, b_{1}, \cdots, b_{d-1}: c_{1}, c_{2}, \cdots, c_{d}\right\}$ is known as the intersection array of $G$. Let $a_{i}=$ $k-b_{i}-c_{i}(i=0,1, \cdots, d)$ be the number of neighbors of $y$ in $N_{i}(x)$ for $d(x, y)=i$. The numbers $a, b_{i}$ and $c_{i}$, are

Revised Manuscript Received on December 16, 2019

* Correspondence Author

M. Saravanan*, Department of Mathematics, Kalasalingam Academy of Research and Education, Krishnankoil,India. Email: msmath.6@ gmail.com

KM. Kathiresan, Director, Centre for Graph Theory, Ayya Nadar Janaki Ammal College, Sivakasi, India. Email: kathir2esan@yahoo.com called the intersection members of $G$. We note that, $b_{0}=k, b_{d}=c_{0}=0, c_{1}=1 . N_{i}(x)$ contains $k_{i}$ points where $k_{0}=1, k_{1}=k, k_{i+1}=\frac{k_{i} b_{i}}{c_{\{i+1\}}}(i=$ $0,1, \cdots, d-1)$ and the number of vertices is $p=1+$ $k_{1}+k_{2}+\cdots+k_{d}$.

\section{PRELIMINARIES}

Consider a square matrix of order $n$ matrix over real numbers of absolute value at most 1 . Then every row vector is of norm at most $\sqrt{n}$ and consequently we have the inequality

$$
|\operatorname{det} A| \leq n^{n / 2}
$$

The inequality (1) is famously known as Hadamard's inequality and the matrices for which equality holds in (1) are known as Hadamard Matrices. For such matrices any two distinct rows must be orthogonal to each other and hence the entries of Hadamard matrices must be \pm 1 . Hence we have the following alternative definition of Hadamard matrix.

Definition 2.1. [6][15] A Hadamard matrix of order $n$ $H=\left[h_{i j}\right]$ is a square matrix of order $n$ such that $h_{i j} \in$ $\{1,-1\}(i, j=1,2, \cdots, n)$ and $H H^{T}=n I$.

Theorem 2.2.[15] Suppose $H$ is a Hadamard matrix of order $n$. Then the value of $n$ should be such that $n=1, n=2$ or $n \equiv 0(\bmod 4)$.

Definition 2.3. [6] Hadamard graph of order $n$ is a distance regular graph with intersection array $\left\{n, n-1, \frac{n}{2}, 1\right.$ : $\left.1, \frac{n}{2}, n-1, n\right\}$.

Hadamard graphs can be constructed from Hadamard matrices as in the following theorem.

Theorem 2.4. Consider a Hadamard matrix $H=\left[h_{i j}\right]$ of order $n$. For $\mathrm{H}$ associate a graph $\mathbb{H}$ whose vertices are the symbols $r_{i}^{+}, r_{i}^{-}, c_{i}^{+}, c_{i}^{-}(i=1$ to $n)$ and whose edges are the 2 sets $\left\{\mathrm{r}_{\mathrm{i}}^{\epsilon}, \mathrm{c}_{\mathrm{j}}^{\eta}\right\}$ with $\epsilon=\eta$ if $h_{i j}=1$ and $\epsilon \neq \eta$ if $h_{i j}=-1$. Then $\mathbb{H}$ is a Hadamard graph of order $n$. Converse is also true. That is, every Hadamard graph arises from a Hadamard matrix in this way.

Here $r$ and $c$ stand for row vertices and column vertices respectively.

Observation 2.5. Hadamard graph of order $n$ is a bipartite $n$ -regular graph on $4 \mathrm{n}$ vertices, where the row vertices and column vertices are the partite sets.

Example 2.6. Hadamard matrix of order 1 is $\mathrm{H}=[1]$ and the corresponding Hadamard graph $\mathbb{H}$ is $2 \mathrm{~K}_{2}$. Which is given in Fig. 2.

Example 2.7. A Hadamard matrix of order 2 is $\left[\begin{array}{cc}1 & -1 \\ -1 & -1\end{array}\right]$ and the corresponding Hadamard graph $\mathbb{H}=\mathrm{C}_{8}$ is given in Fig.3.

\section{Published By:}




\section{A Note on Skew Energy of Hadamard Graph}

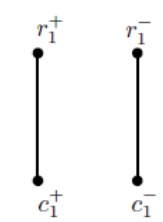

Fig.2. A Hadamard graph of order 1

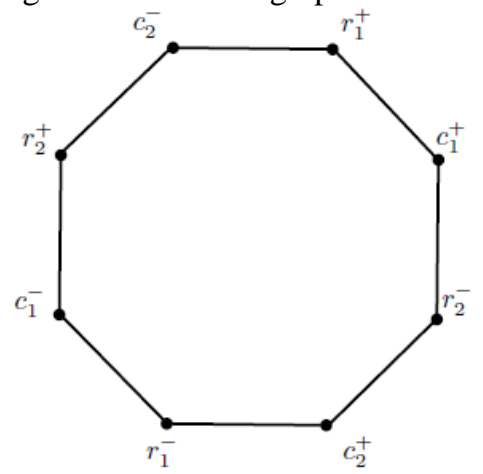

Fig.3. A Hadamard graph of order 2

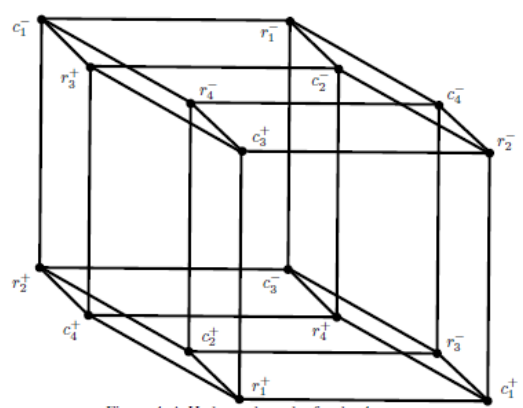

Fig.4. A Hadamard graph of order 4

Example 2.8. A Hadamard matrix of order 4, $\left[\begin{array}{cccc}1 & 1 & 1 & 1 \\ -1 & 1 & -1 & 1 \\ -1 & -1 & 1 & 1 \\ 1 & -1 & -1 & 1\end{array}\right]$ and the corresponding Hadamard graph $\mathbb{H}$, which is Hypercube $Q_{4}$ is given in Fig.4.

Observation 2.9. If $H$ is a Hadamard matrix and $J$ is all one matrix, then the adjacency matrix of $\mathbb{H}$ is

$$
\left[\begin{array}{cc}
0 & A \\
A^{T} & 0
\end{array}\right] \text { where } A=\left[\begin{array}{cc}
\frac{J+H}{2} & \frac{J-H}{2} \\
\frac{J-H}{2} & \frac{J+H}{2}
\end{array}\right] .
$$

Proof. Now positive row vertices are adjacent with positive column vertices, negative row vertices are adjacent with negative column vertices, whenever the corresponding entries of Hadamard matrix is +1 . Positive column vertices are adjacent with negative row vertices and negative column vertices are adjacent with positive row vertices, whenever the corresponding entries of Hadamard matrix is -1 . Hence the adjacency matrix of $\mathbb{H}$ is of the form,

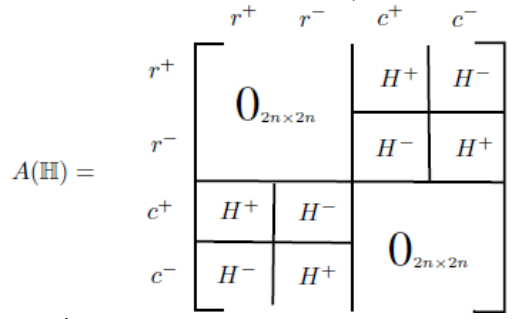

where $H^{+}=\left[h_{i j}^{+}\right]$is an $n \times n$ matrix defined by

$$
h_{i j}^{+}=\left\{\begin{array}{lll}
1 & \text { if } \quad h_{i j}=1 \\
0 & \text { if } \quad h_{i j}=-1
\end{array}\right.
$$

and $H^{-}=\left[h_{i j}^{-}\right]$is an $n \times n$ matrix defined by

$$
h_{i j}^{-}=\left\{\begin{array}{lll}
0 & \text { if } & h_{i j}=1 \\
1 & \text { if } \quad h_{i j}=-1
\end{array}\right.
$$

Now the proof follows as $H^{+}=\frac{J+H}{2}$ and $H^{-}=\frac{J-H}{2}$.

Note 2.10. The existence of Hadamard matrix of order $n$, is still unproved for all $n$. Whenever we write $\mathbb{H}$ is Hadamard graph of order $n$, we assume that the corresponding Hadamard matrix of order $n$ exists. For more on distance regular graphs and Hadamard graphs, we refer [6] .

The spectrum and energy of Hadamard graph were found in [12]

Theorem 2.11. [12] Let $\mathbb{H}$ be a Hadamard graph of order $n$. Then $\operatorname{spec}(\mathbb{H})=\left\{[n]^{1},[\sqrt{n}]^{n},[0]^{2(n-1)},[-\sqrt{n}]^{n},[-n]^{1}\right\}$ and $\mathcal{E}(\mathbb{H})=2 n(1+\sqrt{n})$.

\section{SKEW ENERGY OF A GRAPH}

Let $G$ be a graph on $p$ vertices and $\sigma$ be an orientation that assigns a direction to each edge of $G$. We denote the resulting oriented graph by $G^{\sigma}$. The skew adjacency matrix of the oriented graph $G^{\sigma}$ is the square matrix of order $p, S\left(G^{\sigma}\right)=$ $\left[s_{i j}\right]$, where $s_{i j}=1=s_{-i j}$ whenever $\left(v_{i}, v_{j}\right)$ is an $\operatorname{arc}$ of $G^{\sigma}$ and $s_{i j}=s_{j i}=0$ otherwise. Since the skew adjacency matrix is skew symmetric, the eigen values are all pure imaginary. The skew spectrum $\operatorname{spec}_{S}\left(G^{\sigma}\right)$ of $G^{\sigma}$ is defined as the spectrum of the skew adjacency matrix $S\left(G^{\sigma}\right)$ and consequently the skew energy is defined as the sum of the absolute values of its eigen values, denoted by $\varepsilon_{s}\left(G^{\sigma}\right)$. Skew energy was introduced by Adiga, Balakrishnan and So in [1] and was further studied by many authors [2], [3], [4], [8], [10], [14].

Example 3.1. Consider the path on 4 vertices $P_{4}$ with orientations $\sigma$ and $\tau$ as in the following figure:

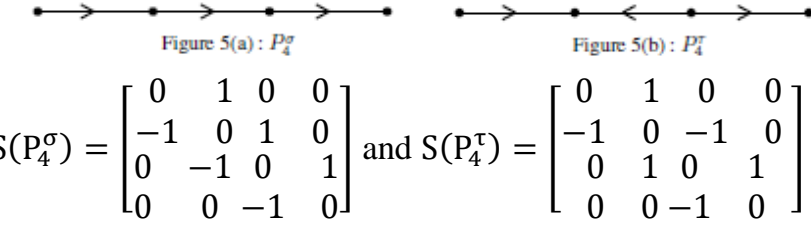

Then the skew spectrum $\operatorname{spec}_{S}\left(P_{4}^{\sigma}\right)=\operatorname{spec}_{S}\left(P_{4}^{\tau}\right)$ $=\left\{\left[ \pm \frac{\sqrt{5}+1}{2} i\right]^{1},\left[ \pm \frac{\sqrt{5}-1}{2} i\right]^{1}\right\}$ and skew energy $\mathcal{E}_{s}\left(P_{4}^{\sigma}\right)=$ $\mathcal{E}_{S}\left(P_{4}^{\tau}\right)=2 \sqrt{5}$.

Now for every edge $r_{i}^{\epsilon} c_{j}^{\eta}$ of Hadamard graph $\mathbb{H}$, it is natural to define an orientation according to the signs $\epsilon$ and $\eta$ as follows: $r_{i}^{\epsilon} \rightarrow c_{j}^{\eta}$ if $\epsilon=\eta$ and $r_{i}^{\epsilon} \leftarrow c_{j}^{\eta}$ if $\epsilon \neq \eta$. We call this orientation as sign orientation and denote it by $\rho$. The resultant oriented graph is denoted by $\mathbb{H}^{\rho}$. We compute the skew spectrum and skew energy of this oriented graph. 
Example 3.2. Oriented Hadamard graph of order 2 is given in Fig.6.

Example 3.3. Oriented Hadamard graph of order 4 is given in Fig.7.

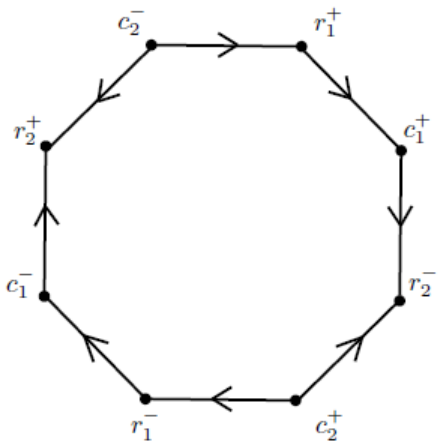

Fig.6. A Hadamard graph of order 2 with sign orientation

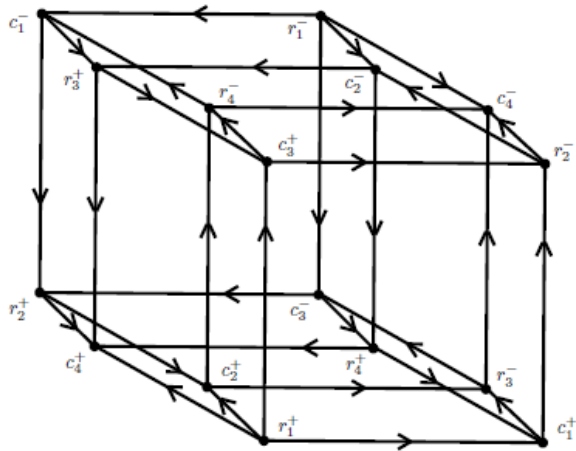

Fig.7. A Hadamard graph of order 4 with sign orientation

We consider an oriented graph $G^{\phi_{1}}$ and a proper subset $U$ of its vertex set. Now reversing the orientations of every arc between $U$ and $\bar{U}$ results in another graph $G^{\phi_{2}}$, where $\bar{U}=$ $V(G) \backslash U$ is the complement of $U$. This process is known as the switch of $G^{\phi_{1}}$ with respect to the subset $U$. If $\phi_{1}$ and $\phi_{2}$ are two orientations of a graph $G$, then $G^{\phi_{1}}$ and $G^{\phi_{2}}$ are said to be switching-equivalent if $G^{\phi_{2}}$ one can be obtained from other by a switch with respect to some subset of $V(G)$.

Consider a bipartite graph $G=G(X, Y)$ with bipartition $V(G)=X \cup Y$. The canonical orientation of $G$ is defined as the orientation that orients all the edges from any one of the partite sets to the other one. In [13] the authors proved that $\operatorname{specs}\left(G^{\sigma}\right)=i \operatorname{spec}(G)$ for the canonical orientation $\sigma$ of $G(X, Y)$. Orientations for which skew spectrum is $i$ multiple of ordinary spectrum have been interestingly studied by many authors. In [8], Cui and Hou conjectured a necessary and sufficient condition for an oriented bipartite graph $G^{\phi}$ to have $\operatorname{specs}\left(G^{\phi}\right)=i \operatorname{spec}(G)$ as $G^{\phi}$ is switching equivalent to $G^{\sigma}$, where $\sigma$ is the canonical orientation of $G$. In [3], Anuradha et al. settled down the conjecture as affirmative.

\section{SKEW ENERGY OF A HADAMARD GRAPH}

In this section, we prove that sign orientation of Hadamard graphs obtained from symmetric Hadamard matrix are switching equivalent to canonical orientation by showing that $\operatorname{spec}_{s}\left(\mathbb{H}^{\rho}\right)=\left\{[i n]^{1},[i \sqrt{n}]^{n},[0]^{2(n-1)},[-i \sqrt{n}]^{n},[-i n]^{1}\right\}$ which is equal to $i \operatorname{spec}(\mathbb{H})$.
Lemma 4.1.[7] Let $A=\left[\begin{array}{ll}A_{0} & A_{1} \\ A_{1} & A_{0}\end{array}\right]$ be a $2 \times 2$ block symmetric matrix. Then the eigen values of $A$ are those of $A_{0}+A_{1}$ and together with $A_{0}-A_{1}$.

Theorem 4.2. Let $H$ be a symmetric Hadamard matrix of order $n, \mathbb{H}$ be the corresponding Hadamard graph of order $n$ and $\mathbb{H}^{\rho}$ be the graph obtained by sign orientation. Then the skew spectrum and skew energy of $\mathbb{H}^{\rho}$ are given by

$$
\begin{gathered}
\operatorname{spec}_{s}\left(\mathbb{H}^{\rho}\right)=\left\{[i n]^{1},[i \sqrt{n}]^{n},[0]^{2(n-1)},[-i \sqrt{n}]^{n},[-i n]^{1}\right\} \\
\text { and } \mathcal{E}_{s}\left(\mathbb{H}^{\rho}\right)=2 n(1+\sqrt{n}) .
\end{gathered}
$$

Proof. Now the arcs are from positive row vertices to positive column vertices, negative row vertices to negative column vertices, positive column vertices to negative row vertices and negative column vertices to positive row vertices, whenever the corresponding vertices are adjacent in the underlying undirected Hadamard graph $\mathbb{H}$. Therefore the adjacency matrix of $\mathbb{H}^{\rho}$ is

$$
\begin{aligned}
A\left(\mathbb{H}^{\rho}\right) & =\left[\begin{array}{cc}
0_{2 n \times 2 n} & \left.\begin{array}{cc}
\frac{H+J}{2} & \frac{H-J}{2} \\
\frac{H-J}{2} & \frac{H+J}{2}
\end{array}\right) \\
-\left(\begin{array}{cc}
\frac{H+J}{2} & \frac{H-J}{2} \\
\frac{H-J}{2} & \frac{H+J}{2}
\end{array}\right) & 0_{2 n \times 2 n}
\end{array}\right] \\
& =\left[\begin{array}{cc}
0 & 1 \\
-1 & 0
\end{array}\right] \otimes\left[\begin{array}{cc}
\frac{H+J}{2} & \frac{H-J}{2} \\
\frac{H-J}{2} & \frac{H+J}{2}
\end{array}\right] \\
& = \pm i \times \operatorname{spec}\left[\begin{array}{cc}
\frac{H+J}{2} & \frac{H-J}{2} \\
\frac{H-J}{2} & \frac{H+J}{2}
\end{array}\right] .
\end{aligned}
$$

Now by Lemma 4.'1, spec $\left[\begin{array}{cc}\frac{H+J}{2} & \frac{H-J}{2} \\ \frac{H-J}{2} & \frac{H+J}{2}\end{array}\right]=\operatorname{spec}(H) \cup$ $\operatorname{spec}(J)$. Then the proof follows from $\operatorname{spec}(H)=$ $\left\{[\sqrt{n}]^{\frac{n}{2}},[-\sqrt{n}]^{\frac{n}{2}}\right\}$, since $H$ is symmetric and $\operatorname{spec}(J)=$ $\left\{[n]^{1},[0]^{n-1}\right\}$.

\section{ACKNOWLEDGMENT}

The first author thanks National Board for Higher Mathematics, DAE, India for supporting him by NBHM Ph.D. fellowship (Grant number 2/39(2)/2009/NBHM/R\& D-II/1941), since a part of this work is done by him during his Ph.D.

\section{REFERENCES}

1. C. Adiga, R. Balakrishnan and W. So, The skew energy of a digraph Linear Algebra Appl., 432 (2010), 1825-1835.

2. A. Anuradha and R. Balakrishnan, Skew spectrum of the Cartesian product of an oriented graph with an oriented hypercube. In Combinatorial Matrix Theory and Generalized Inverses of Matrices, Eds. R. B. Bapat, S. J. Kirkland, K. M. Prasad, S. Puntanen, Springer, (2013), 1-12. 


\section{A Note on Skew Energy of Hadamard Graph}

3. A. Anuradha, R. Balakrishnan, X. Chen, X. Li, H. Lian and W. So, Skew spectra of oriented bipartite graphs, The Electronic J. Combin. 20(4) (2013), \#18.

4. A. Anuradha, R. Balakrishnan, X. Chen, X. Li, H. Lian and W. So, Skew spectra of graphs without even cycles, Linear Algebra Appl., 444 (2014), 67-80.

5. R. Balakrishnan, The energy of a graph, Linear Algebra Appl., 387 (2004), 287-295.

6. A. E. Brouwer, A. M. Cohen and A. Neumair, Distance Regular graphs, Springer - verlag, NewYork, (1989).

7. D. Cvetkovic, M. Doob and H. Sachs, Spectra of Graphs : Theory and Application, Academic Press, New York, (1980).

8. D. Cui and Y. Hou, On the skew spectra of Cartesian products of graphs, The Electronic J. Combin., 20(2) (2013), \#P19.

9. I. Gutman, in The Energy of a Graph: Old and New Results, in: A.Beten, A. Kohnert, R. Laue, A. Wassermann(eds.) Algebraic Combinatorics and Applications, Springer - verlag, Berlin, (2001), 196-211.

10. X. Li and H. Lian, A survey on the skew energy of oriented graphs. Available at arXiv: 1304.5707

11. X. Li, Y. Shi and I. Gutman, Graph Energy, Springer, New York, 2012.

12. M. Saravanan and KM. Kathiresan, On the Energy of Hadamard Graph, Bull. Inst. Combin. Appl., 76 (2016), 16-28.

13. B. Shader and W. So. Skew spectra of oriented graphs. The Electronic J Combin.,16 (2009), 1-6.

14. G-X. Tian, On the skew energy of orientations of hypercubes. Linear Algebra Appl., 435 (2011), 2140-2149.

15. J. H. van Lint and R. M. Wilson, A course in combinatorics, Cambridge University Press, (2001)

\section{AUTHORS PROFILE}

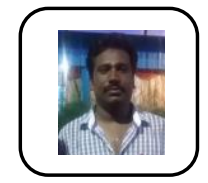

M. Saravanan has done his Ph.D. in Spectral Graph theory.

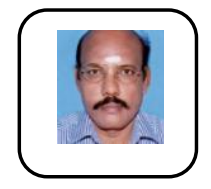

KM. Kathiresan had done his Ph.D. in Graph theory. He has published more than 80 articles in reputed journals. He has served as reviewer in many reputed journals and he is the member of many academic societies. Also he has received "TANSA - 2012 Award (Tamilnadu Scientist Award)" awarded by TNSCST. 